\title{
Seguimiento del prematuro/gran prematuro en Atención Primaria
}

\author{
S. Caserío Carbonero, CR. Pallás Alonso \\ Servicio de Neonatología, Hospital 12 de Octubre. Madrid. España.
}

Rev Pediatr Aten Primaria. 2009; I I (Supl I 7):s443-s450

Sonia Caserío Carbonero, caseriocs@hotmail.com

Resumen

Dado que la prematuridad es uno de los principales problemas sanitarios de los países desarrollados, este taller tiene como objetivo dotar al equipo de Atención Primaria de los conocimientos y herramientas necesarias, para hacerse cargo de forma conjunta y coordinada con el equipo hospitalario, de los programas de seguimiento de los niños con una edad gestacional menor de 32 semanas o con un peso al nacimiento inferior a $1.500 \mathrm{~g}$. Para ello, el pediatra de Atención Primaria debe conocer cómo es el desarrollo habitual de estos niños, las características de su evolución, las variantes que presentan con respecto a la normalidad, los problemas que pueden aparecer y los signos de alarma que pueden ayudar a identificarlos con precocidad. Hay que asegurarse de que estos niños estén en un programa de seguimiento -que de forma ideal debería extenderse hasta la adolescencia-y acudan a los controles; también se debe prestar apoyo a la familia y evitar repeticiones innecesarias de pruebas. En los programas de seguimiento se realizan múltiples cribados y recomendaciones con objeto de mejorar en lo posible la evolución a medio y largo plazo de los niños.

Recomendamos la lectura del texto completo del "Programa de actividades preventivas y de promoción de la salud para niños prematuros con una edad gestacional menor de 32 semanas o un peso inferior a 1.500 g. Del alta hospitalaria a los 7 años" de C. Pallás Alonso para PrevInfad, en el que está basado este taller y texto. Disponible en www.aepap.org/previnfad/pdfs Iprevinfad_menor32-1500.pdf.

Palabras clave: Prematuridad, Seguimiento, Prevención.

\section{Abstract}

Given that prematurity is one of the principal health problems in developed countries, the aim of this workshop is to provide the Primary Care team with the knowledge and the tools necessary to be able to be responsible, jointly and coordinating with the Hospital team, for follow up programs of children with a gestational age of less than 32 weeks or with a birth weight of less than 1500 g. To do this, the Primary Care paediatrician must know what is the usual development in these children, the characteristics of their progress, the variations within normal that can be seen, the problems that can occur, and the alarm signs that may help to identify them

Las autoras declaran no presentar posibles conflictos de intereses en relación con la preparación y publicación de este artículo. 
early. You must ensure that these children are in a follow up program - which ideally is extended until adolescence - and that they attend reviews. Support must also be provided for the family and avoid repeating unnecessary tests. Multiple screening and recommendations are included in follow up programs, with the purpose of improving the progress of the children in the medium to long term.

We recommend reading the complete text of the "Preventive Activities Program and health promotion for premature children with a gestational age of less than 32 weeks or a birth weight of less than $1500 \mathrm{~g}$. From hospital discharge to 7 years old" by C. Pallás Alonso for Prevlnfad, on which this workshop and text is based. (Available at: www.aepap.org/previn $\mathrm{fad} /$ pdfs/previnfad_menor32-1500.pdf.

Key words: Prematurity, Follow up, Prevention.

\section{Introducción}

La prematuridad es uno de los principales problemas sanitarios de los países desarrollados, aunque por motivos no bien conocidos tenga menos visibilidad que otros problemas de salud. Entre un 8-10\% de los nacimientos ocurren antes de la 37 semana de gestación y justifican el $75 \%$ de la mortalidad perinatal y el $50 \%$ de la discapacidad en la infancia. A pesar de todos los esfuerzos realizados en el ámbito clínico y de investigación, la frecuencia de la prematuridad se está incrementando. Entre los factores implicados se encuentran las técnicas de reproducción asistida ${ }^{1}$, el incremento de la edad materna, ciertas condiciones laborales y situaciones de estrés. Además, recientemente se ha publicado que las mujeres que fueron prematuras presentan mayor riesgo de tener hijos prematuros ${ }^{2}$.

Los avances terapéuticos en las unidades de cuidados intensivos neonata- les han conseguido mejorar la supervivencia de los recién nacidos prematuros de muy bajo peso al nacer. El cambio en el manejo obstétrico y el avance en los cuidados perinatales, fundamentalmente el empleo de los corticoides prenatales ${ }^{3}$ y la administración del surfactante exógeno $0^{4,5}$, han contribuido a un claro aumento de la supervivencia de los prematuros de muy bajo y de extremo bajo peso al nacer. Sin embargo, surge la preocupación de si este incremento en la supervivencia no conllevará un aumento de la discapacidad.

La tasa de supervivencia aislada no es el mejor indicador de calidad de una unidad neonatal, es fundamental considerar también la tasa de supervivencia sin discapacidad. Así a los neonatólogos, en un principio, les preocupaba sobre todo la supervivencia, pero muy pronto se fue consciente de que la mortalidad era solo una parte del efecto de la prematuridad. Se demostró un mayor 
riesgo en estos pacientes de parálisis cerebral, ceguera, retraso mental y sorde$\mathrm{ra}^{6,7}$. Más recientemente también se ha identificado peor rendimiento escolar por dificultades en el aprendizaje, problemas de atención, de coordinación visomotora, problemas emocionales y de integración social ${ }^{8-12}$.

Las implicaciones médicas, éticas y económicas derivadas del tratamiento de este grupo de niños son siempre objeto de debate.

En las unidades neonatales se ha empezado a prestar atención a otro tipo de cuidados, llamados cuidados centrados en el desarrollo, que no se centran tanto en curar patologías como en conseguir el adecuado desarrollo neurológico y emocional del niño ${ }^{13}$. Está cambiando la política de entrada de los padres en las unidades y de la implicación de estos en los cuidados de sus hijos. Se intenta cuidar el ambiente, disminuyendo los estímulos agresivos y se trata cuidadosamente el dolor, para que el sistema nervioso madure de forma organizada como lo habría hecho en el útero materno ${ }^{14,15}$.

Tras el alta, sin duda, los niños con una edad inferior a 32 semanas o con un peso de nacimiento inferior a $1.500 \mathrm{~g}$, precisan de una serie de cribados y controles sistematizados, ya que están en mayor riesgo de presentar problemas en el desarrollo, ya sean de crecimiento, neurológicos, sensoriales o psicológicos. Por otro lado, para los padres y madres, el ingreso tan prologando de su hijo prematuro es un factor muy estresante que conlleva posteriormente un mayor riesgo de problemas psicológicos también en ellos y en muchas ocasiones el proceso de crianza se ve alterado ${ }^{16}$.

\section{Contenido}

\section{Términos}

- Recién nacido (RN) a término: el nacido entre la 37 semana de edad gestacional (EG) y la 41 semana más 6 días.

- RN pretérmino: el nacido antes de la 37 semana EG.

- RN postérmino: el nacido después de la 41 semana más 6 días.

- Gran prematuro o muy prematuro: el nacido antes de la 32 semana EG.

- Prematuro extremo: el nacido antes de la 28 semana EG.

- Prematuro tardío: el nacido con 34 semanas de EG o más. No utilizar la denominación RN casi a término.

- Edad corregida: sería la edad que tiene el niño si hubiera nacido el día que cumplía la 40 semana de 
EG. Se corrige la edad para la valoración del peso, talla, perímetro cefálico, cociente de desarrollo y adquisiciones motoras hasta los 2 años cumplidos de edad corregida.

- RN de bajo peso: peso al nacimiento menor de $2.500 \mathrm{~g}$ independientemente de la EG.

- RN de muy bajo peso: peso al nacimiento menor de $1.500 \mathrm{~g}$ independientemente de la EG.

- RN de bajo peso extremo: peso al nacimiento menor de $1.000 \mathrm{~g}$ independientemente de la EG.

- RN de peso adecuado para la EG: peso al nacimiento entre el percentil 10 y 90 para su EG.

- RN de bajo peso para la EG: peso al nacimiento por debajo del percentil 10 para su EG.

- RN de peso elevado para la EG: peso al nacimiento por encima del percentil 90 para su EG.

\section{Recomendaciones para el pediatra}

- Poseer un adecuado conocimiento del programa de seguimiento en el que está el niño incluido.

- Asegurarse de que el niño acude a las revisiones y se le realizan los controles y pruebas necesarios.

- Debe conocer el patrón de crecimiento normal de estos niños, que es diferente al de los niños nacidos a término y del de los prematuros de mayor EG y peso. Mientras que no se disponga de otros estándares, se recomienda la utilización de los estándares de la $\mathrm{OMS}^{17}$ (según la edad corregida).

- Seguimiento exhaustivo del crecimiento tras el alta. Conociendo que el crecimiento insuficiente se asocia con problemas en el neurodesarrollo a medio y largo plazo ${ }^{18-20}$, mientras que la excesiva ganancia de peso se asocia con un aumento del riesgo cardiovascular, obesidad y diabetes en la edad adulta ${ }^{21-23}$. Valorar como crecimiento adecuado cuando las curvas de peso y talla siguen una línea casi paralela a la de los percentiles, pero con tendencia progresiva a acercarse a las líneas normales. Vigilando que el incremento de peso y talla sea armónico.

- Apoyar y promocionar la lactancia materna tras el alta. Puesto que además de los beneficios obtenidos durante el ingreso hospitalario, mejora el cociente de desarrollo y disminuyen las tasas de reingreso ${ }^{24,25}$.

- Conocer el método canguro (contacto piel con piel), puesto que mientras que el niño lo tolera (has- 
ta las $39-40$ semanas) incrementa la producción de leche y favorece el vínculo ${ }^{26}$.

- Suplementar desde los 15 días hasta el año con 400 UI/día vitamina $D^{27,28}$. Vigilar la aparición de raquitismo, se recomienda determinación de fosfatasa alcalina a partir del mes después del alta y si está elevada confirmar el diagnóstico radiológicamente.

- Suplementar con 4 mg/kg/día (máximo $15 \mathrm{mg} /$ día) de hierro (sal ferrosa) desde el mes de edad hasta la introducción de la alimentación complementaria con alimentos ricos en hierro ${ }^{29}$. Se puede hacer un control de hemograma y metabolismo férrico a los 3 meses de edad corregida.

- Los niños de bajo peso para EG que a los 2-3 años tienen una talla por debajo de dos desviaciones estándar deben enviarse para valorar administración de hormona de crecimiento ${ }^{30,31}$.

- Se realizará una detenida evaluación motora al menos dos veces en el primer año de vida, aunque aparentemente el desarrollo sea adecuado.

- Todos los niños con riesgo de problemas de desarrollo motor que no estén incluidos en un programa de seguimiento y en los que se detecte alguna alteración motora deben remitirse a Atención Temprana.

- Utilización de los algoritmos propuestos por SCPE, para excluir o diagnosticar parálisis cerebral ${ }^{32}$.

- Utilización de escalas de función motora para evaluar a los niños en los que se sospeche o se haya confirmado una alteración motora.

- Confirmará que se haya realizado y completado el cribado para la retinopatía de la prematuridad. Si no fuera así, lo derivará inmediatamente a un oftalmólogo experto.

- A pesar de que los niños no tengan patología oftalmológica grave o neurológica, se recomendará una evaluación oftalmológica antes de los 3 años ${ }^{33}$.

- Debe remitirse al oftalmólogo cualquier niño en el que el pediatra detecte una alteración o sea referida por los padres ${ }^{34}$.

- Los niños con antecedente de retinopatía grado 3 o que hayan requerido tratamiento y los que tengan patología neurológica, precisan seguimiento oftalmológico hasta la adolescencia ${ }^{33}$.

- Recordar que casi el 20\% de los niños muy prematuros abandonan 
los programas de seguimiento y estos niños tienen peor evolución que los que continúan en el programa $^{35}$.

\section{Bibliografía}

1. Goldenberg RL, Culhane JF, lams JD, Romero R. Epidemiology and causes of preterm birth. Lancet. 2008;371:75-84.

2. Swamy GK, Osbye T, Skjaerven R. Association of preterm birth with long term survival, reproduction and next generation preterm birth. JAMA. 2008;299:1429-36.

3. National Institutes of Health Consensus Development Conference: Effect of corticosteroids for fetal maduration on perinatal outcomes. Am J Obstet Gynecol. 1995;173:246-8.

4. Collaborative European Group. Surfactant replacement therapy for severe neonatal respiratory distress syndrome: an internacional randomized clinical trial. Pediatrics. 1988;82:683-91.

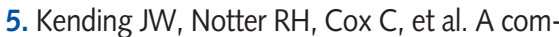
parison of surfactant as immediate prophylaxis and as rescue therapy in newborn of less than 30 week's gestation. N Eng J Med. 1991;324:865-87.

6. Sutton L, Bajuk B. Population based study of infants born at less than 28 weeks gestation in New South Wales, Australia, in 1992-3. New South Wales Neonatal Intensive Care Unit Study Group. Paediatr Perinat Epidemiol. 1999;13:288301.

7. Finnström O, Otterblad Olausson $P$, Sedin $G$, Serenius F, Svenningsen N, Thiringer $K$, et al. Neurosensory outcome and growth at three years in extremely low birthweight infants: follow-up results from Sweedish national prospective study. Acta Paediatr. 1998;87:1055-60.

8. Delobel-Ayoub $M$, Kaminski $M$, Marret $S$, Burquet A, Marchand L, N'Guyen S, et al. Beha- vioural outcome at 3 years of age in very preterm infants: the EPIPAGE study. Pediatrics. 2006;117: 1996-2005.

9. Reijneveld SA, De Kleine MJK, Van de Baar AL, Kollée LA, Verhaak CM, et al. Behavioural and emotional problems in very preterm and very low birthweight infants at age 5 years. Arch Dic Child Fetal Neonatal Ed. 2006;91:F423-8.

10. Hille ET, Den Ouden Al, Saigal S, Wolke D, Lambert $M$, Whitaker $A$, et al. Behavioural problems in children who weight 1000 gr or less at birth in four countries. Lancet. 2001;357:1641-3.

11. Elgen I, Sommerfelt K, Markestad T. Population based, controlled study of behavioural problems and psychiatric disorders in low birthweight children at 11 years of age. Arch Dis Child Fetal Neonatal Ed. 2002;87:F128-32.

12. Saigal S, Pinelli J, Hoult L, Kim MM, Boyle M. Psychopatology and social competencies of adolescents who were extremely low birth weight. Pediatrics. 2003;111:969-75.

13. Sizun J, Westrup B. Early development care for preterm neonates: a call for more research. Arch Dis Child Fetal Neonatal Ed. 2004;89:F384-8.

14. Perapoch J, Pallás CR, Linde MA, et al. Cuidados centrados en el desarrollo. Situación en las unidades de neonatología de España. An Pediatr (Barc). 2006;64:132-9.

15. Pallás CR, Arriaga M. Nuevos aspectos entorno a la prematuridad. Evid Pediatr. 2008;4:26.

16. Pallás CR. Programa de actividades preventivas y de promoción de la salud para niños prematuros con una edad gestacional menor de $32 \mathrm{se}$ manas o un peso inferior a 1.500 gramos. Del alta hospitalaria a los 7 años. En PrevInfad (AEPap)/ 
PAPPS Infancia y adolescencia [en línea]. Octubre 2008 [consultado el 20/10/2009]. Disponible en www.aepap.org/previnfad/pdfs/previnfad_me nor32-1500.pdf

17. WHO Multicentre Growth Reference Study Group. WHO Child Growth Standards based on lenght/weight, weight and age. Acta Paediatr Suppl. 2006;450:76-85.

18. Latal-Hajnal B, Von Siebenthal $K$, Kovari $H$, et al. Postnatal growth in VLBW infants: significant association with neurodevelopmental outcome. J Pediatr. 2003;143:163-70.

19. Cooke RWI, Foulder-Hughes L. Growth impairment in the very low preterm and cognitive and motor performance at 7 years. Arch Dis Child. 2003;88:482-7.

20. Casey PH, Whiteside-Mansell L, Barrett K. Impact of prenatal and postnatal growth problems in low birth weight preterm infants on school age outcomes: an 8 years longitudinal evaluation. Pediatrics. 2006;118:1078-86.

21. Barker DJP, Osmond C, Forsen TJ. Trajectories of growth among children who have coronary events as adults. N Engl J Med. 2005;353:1802-9.

22. Hovi $P$, Andersson S, Eriksson JG. Glucose regulation in young adults with very low birth weight. N Engl J Med. 2007;356:2053-63.

23. Hofman PL, Regan F, Jackson WE. Premature birth and later insulina resistance. N Engl J Med. 2004;351:2179-86.

24. Vohr BR, Poindexter BB, Dusick AM; NICHD Neonatal Research Network. Beneficial effects of breast milk in the neonatal intensive care unit on the developmental outcome of extremely low birth weight infants at 18 months of age. Pediatrics. 2006;118:e115-23.

25. Vohr BR, Poindexter BB, Dusick AM; National Institute of Child Health and Human Development National Research Network. Persistent beneficial effects of breast milk ingested in the neonatal intensive care unit on outcomes of extremely low birth weight infants at 30 months of age. Pediatrics. 2007;120:e953-9.

26. Charpak NM, Ruiz-Peláez JG, Figueroa de CZ, Charpak Y. A randomized, controlled trial of Kangaroo Mother Care: results of follow up at 1 year of corrected age. Pediatrics. 2001;108: 1072-9.

27. Munns C. Prevention and treatment of infant and childhood vitamin D deficiency in Australia and New Zeland: a consensus statement. Med J Aust. 2006;185:268-72.

28. Pallás Alonso CR. Vitaminas y oligoelementos. En: Recomendaciones Previnfad/PAPPS [en línea] [consultado el 20/10/2009]. Disponible en www.aepap.org/previnfad/vitaminas.htm [actualizado el 12/2006].

29. Schandler RJ. Post-discharge nutrition for the preterm infant. Acta Paediatr Suppl. 2005; 94:68-73.

30. Lee PA, Chernausek SD, Hokken-Koelega AC, Czernichow. International Small for Gestational Age Advisory Board consensus development conference statement: management of short children born small for gestational age. Pediatrics. 2003;111:1253-61.

31. Clayton PE, Cianfarani S, Czenirchow P, Johannsson, Rapaport R, Rogol A. Management of the child born small for gestational age through to adulthood: a consensus statement of the International Societies of Paediatric Endocrinology and the Growth Hormone Research Society. J Clin Endocrinol Metab. 2007;92:804-10.

32. Surveillance of Cerebral Palsy in Europe: a collaboration of cerebral palsy surveys and registers. Surveillance of Cerebral Palsy in Europe (SCPE). Dev Med Child Neurol. 2000;42(12): 816-24.

33. O'Connor AR, Stewart CE, Singh J, Fielder AR. Do infants of birth weight less than $1.500 \mathrm{~g}$ re- 
quire additional long term ophthalmic follow up? Br Ophthalmol. 2006;90:451-5.

34. Martín Begue N, Perapoch López J. Retinopatía de la prematuridad: incidencia, gravedad y seguimiento. An Pediatr. 2003;58:156-61.
35. López Maestro M, Pallás CR, de la Cruz J, Pérez I, Gómez E, de Alba C. Abandonos en el seguimiento de recién nacidos de muy bajo peso y frecuencia de parálisis cerebral. An Esp Pediatr. 2002;57(4):354-60. 